\title{
ARTICLE OPEN Secreted frizzled related-protein 2 (Sfrp2) deficiency decreases adult skeletal stem cell function in mice
}

Luis Fernandez de Castro ${ }^{1}{ }^{1 凶}$, Brian J. Sworder ${ }^{1,2}$, Byron Mui ${ }^{1}$, Kathryn Futrega ${ }^{1}$, Agnes Berendsen ${ }^{1}$, Matthew D. Phillips ${ }^{1}$, Nathan J. Burbach ${ }^{1,3}$, Natasha Cherman ${ }^{1}$, Sergei Kuznetsov ${ }^{1}$, Yankel Gabet ${ }^{4}$, Kenn Holmbeck ${ }^{1}$ and Pamela G. Robey ${ }^{1 凶}$

In a previous transcriptomic study of human bone marrow stromal cells (BMSCs, also known as bone marrow-derived "mesenchymal stem cells"), SFRP2 was highly over-represented in a subset of multipotent BMSCs (skeletal stem cells, SSCs), which recreate a bone/marrow organ in an in vivo ectopic bone formation assay. SFRPs modulate WNT signaling, which is essential to maintain skeletal homeostasis, but the specific role of SFRP2 in BMSCs/SSCs is unclear. Here, we evaluated Sfrp2 deficiency on BMSC/SSC function in models of skeletal organogenesis and regeneration. The skeleton of Sfrp2-deficient (KO) mice is overtly normal; but their BMSCs/SSCs exhibit reduced colony-forming efficiency, reflecting low SSC self-renewal/abundancy. Sfrp2 KO BMSCs/SSCs formed less trabecular bone than those from WT littermates in the ectopic bone formation assay. Moreover, regeneration of a cortical drilled hole defect was dramatically impaired in Sfrp2 KO mice. Sfrp2-deficient BMSCs/SSCs exhibited poor in vitro osteogenic differentiation as measured by Runx2 and Osterix expression and calcium accumulation. Interestingly, activation of the Wnt co-receptor, Lrp6, and expression of Wnt target genes, Axin2, C-myc and Cyclin D1, were reduced in Sfrp2-deficient BMSCs/SSCs. Addition of recombinant Sfrp2 restored most of these activities, suggesting that Sfrp2 acts as a Wnt agonist. We demonstrate that Sfrp2 plays a role in self-renewal of SSCs and in the recruitment and differentiation of adult SSCs during bone healing. SFRP2 is also a useful marker of BMSC/SSC multipotency, and a factor to potentially improve the quality of ex vivo expanded BMSC/SSC products.

Bone Research (2021)9:49

; https://doi.org/10.1038/s41413-021-00169-7

\section{INTRODUCTION}

Bone marrow stromal cells (BMSCs, also referred to as bone marrow-derived "mesenchymal stem cells"), are a population of non-hematopoietic, rapidly adherent fibroblastic cells first described by Friedenstein and Owen [reviewed in refs. ${ }^{1,2}$ ] Nonclonal strains of BMSCs generate cartilage in an in vitro cell pellet assay, as well as osteogenic cells, hematopoiesis-supportive stroma and marrow adipocytes upon in vivo transplantation with appropriate scaffolds, based on the presence of a subset of skeletal stem cells (SSCs) within bone marrow stromal cells (bmSSCs). ${ }^{3}$ These cells are anatomically, phenotypically and functionally distinct from SSCs within the developing growth plate (gpSSCs), and in the periosteum $\left(\right.$ pSSCs) ${ }^{4-8}$ (reviewed in ref. ${ }^{9,10}$ ).

When a single-cell suspension of freshly isolated human bone marrow is plated at low density, a number of individual BMSCs/ SSCs, known as colony-forming unit-fibroblasts (CFU-Fs), rapidly adhere and proliferate, generating colonies. When single colonyderived strains (SCDSs, initiated by a single CFU-F) were transplanted in vivo to form ectopic ossicles, $12.5 \%$ of them were able to generate a complete bone-marrow organ and were designated multipotent SCDSs (M-SCDSs) containing bmSSCs. The remaining strains either formed bone tissue but did not support hematopoiesis $(66.7 \%, \mathrm{~B}-\mathrm{SCDSs})$, or formed fibrous tissue $(20.8 \%, F-S C D S s)$. The transcriptomic profile of aliquots of the M-SCDSs and F-SCDSs cultures used for in vivo transplantation were compared, and SFRP2 gene was highly and consistently over-represented in M-SCDSs, suggesting its potential role in SSCs. ${ }^{11}$

SFRP2 is one of five members of the Secreted Frizzled-Related Protein (SFRP) family [FRZB, SFRP1, 2, 4, 5 (also known as COMP)]. SFRPs share two conserved domains separated by a short linker region: a N-terminal Cysteine Rich Domain (CRD), with homology to the WNT-binding site in Frizzled, and a C-terminal Netrin-related Domain (NTR) with homology to Laminin. ${ }^{12}$ Members of the SFRP family are reported to be modulators of the Wnt signaling pathway, which has emerged as a critical component in bone modeling, remodeling and regeneration (reviewed in ref. ${ }^{13-15}$ ) Reduction in WNT signaling can result in loss of bone mass, ${ }^{16,17}$ while excessive activation has the opposite effects. ${ }^{18-20}$ Indeed, WNT pathway activation improves healing of transcortical defects through SSC differentiation into osteoblasts. ${ }^{21,22}$ Fine-tuning of this pathway is controlled by combinations of Frizzled receptors and co-receptors; as well as factors that bind to WNT and/or its receptors, including SFRPs, and an ever-increasing list of ligands.

Previous studies have reported negative effects of SFRP2 on WNT signaling in osteoblast differentiation and bone formation $\left[\right.$ e.g., ${ }^{23,24}$ ] However, many of these studies solely utilized cell line models. One study of Sfrp2-deficient (KO) mice showed subtle

\footnotetext{
'Skeletal Biology Section, National Institute of Dental and Craniofacial Research, National Institutes of Health, Department of Health and Human Services, National Institutes of Health, Bethesda, MD, USA; ${ }^{2}$ Department of Molecular Medicine, Boston University, Boston, MA, USA; ${ }^{3}$ School of Dentistry, University of Minnesota-Twin Cities, Minneapolis, MN, USA and ${ }^{4}$ Department of Anatomy and Anthropology, Sackler Faculty of Medicine, Tel Aviv University, Tel Aviv-Yafo, Israel

Correspondence: Luis Fernandez de Castro (luis.fernandezdecastrodiaz@nih.gov) or Pamela G. Robey (pamela.robey@nih.gov)
}

Received: 18 February 2021 Revised: 21 May 2021 Accepted: 27 June 2021

Published online: 02 December 2021 
differences in long bone length in the distal portion of the appendicular skeleton, leading to mild brachydactyly, the cause of which was determined to be decreased chondrocyte proliferation and delayed maturation. ${ }^{25}$ However, a comprehensive skeletal analysis was not performed to determine other potential changes in the skeleton of Sfrp2 KO mice.

Although previously regarded as inhibitors of WNT signaling, the action of SFRPs is now recognized to be more complex. SFRPs may operate as either WNT inhibitors or activators depending on the cell type, receptor expression pattern, and the local presence of other WNT-related or unrelated factors. ${ }^{26-28}$ Motivated by the high SFRP2 expression observed in human multipotent BMSCs/ SSCs, we obtained Sfrp2 KO mice to investigate the role of this gene in postnatal BMSCs/SSCs during bone regeneration, and its modulation of the Wnt pathway during their differentiation.

\section{RESULTS}

Sfrp2 expression is enriched in Lepr $^{+}$bone marrow-derived stromal stem/progenitor cells in mice

We analyzed a publicly available single-cell RNA-seq datasets ${ }^{29}$ to determine the expression of Sfrp2 in the mouse nonhematopoietic bone marrow compartment. These datasets were generated using cells that were isolated from lineage-specific Cre-transgenic mice crossed with a knock-in reporter strain, Rosa26 locus (LoxP-tdTomato). The datasets included cells marked by the expression of VE-Cad, Lepr and the Col $2.3 \mathrm{~kb}$ promoter (Col1a1) to identify vascular, bone marrow-derived stem/progenitor cells (termed "mesenchymal stromal cells" by the authors), and osteoblasts at steady state, respectively (see ${ }^{29}$ for experimental details). We performed our own independent analysis of the data using the Seurat package in R. We found that $\mathrm{Sfrp}^{+}$cells were localized within the Lepr $^{+}$population, specifically in cluster 2 (average log2FC of 0.89 and $P$-adjusted value of $8.7 \times 10^{-163}$ ) (Fig. 1). These results indicate that, as in human bone marrow, Sfrp2 is expressed by a subset of bone marrow-derived skeletal stem/progenitor cells.

\section{Sfrp2 KO mice exhibit normal bone structure and morphometric features}

The skeletal phenotype of Sfrp2 KO mice was first characterized using a variety of imaging modalities. In addition to the mild brachy-syndactyly already described in this model (not shown), ${ }^{25}$ high-definition $\mathrm{X}$-rays of these mice did not show any additional skeletal phenotype at 2 months of age (Fig. 2a). Bone mineral density measured by X-ray absorptiometry (DEXA) was not altered (data not shown). Femoral analyses by $\mu \mathrm{CT}$ and histomorphometry at 3 and 11 months were also unremarkable and did not discriminate WT from Sfrp2 KO mice (Supplementary Fig. 1). Cranial anatomy of 3 -month-old animals analyzed by $\mu C T$ was likewise unremarkable (Supplementary Fig. 2A). Calvariae were stained for TRAP activity as an approximation of osteoclastic activity. The cranial sutures [a common area of abnormal bone resorption in mice ${ }^{30}$ ] were not affected by the absence of Sfrp2 (Supplementary Fig. 2B). To better examine changes in soft tissues in relationship to mineralized tissues, WT and Sfrp2 KO femora were examined histologically. Again, there was no histological evidence of changes in Sfrp2 KO femora compared with WT femora at 3 months and 11 months of age (Fig. 2b, c).

\section{Sfrp2-deficient BMSCs/SSCs have reduced colony-forming} efficiency (CFE)

An initial characterization of the cellular phenotype of the Sfrp2 $\mathrm{KO}$ mice was the determination of colony-forming efficiency (CFE) of single-cell suspensions of bone marrow, a measure of the number of colony-forming units-fibroblasts (CFU-Fs). The number of CFU-Fs in bone marrow is currently the closest approximation of the number of bmSSCs present in the BMSC population. ${ }^{31}$ When cultured at clonal density, bone marrow cells from Sfrp2 KO mice showed a pronounced decrease ( 40\%) in the number of colonies derived from single CFU-Fs after 14 days of culture (Fig. 3a).

Sfrp2 KO BMSCs have a decreased ability to form a bone/marrow organ de novo in vivo

A well-established in vivo assay that measures the intrinsic ability of bmSSCs within BMSC populations to form a bone/marrow organ is by transplantation of the cells along with an appropriate scaffold into a subcutaneous pocket in immunocompromised mice (in vivo ectopic ossicle). ${ }^{32}$ Establishment of a complete bone/ marrow organ by transplanted cells is completely dependent on the presence of the bmSSC within the transplanted BMSC population ${ }^{2}$ (Fig. 3b). For this purpose, WT and Sfrp2 KO BMSCs/ SSCs were established in culture. No differences in growth rate were observed between the two genotypes (data not shown). At passage 2 or 3 , the cells were depleted of $C D 45^{+} / C D 11 b^{+}$ hematopoietic cells before being absorbed into Gelfoam ${ }^{T M}$ (gelatin) sponges and transplanted into subcutaneous pockets in immunocompromised mice. Eight weeks later, the ectopic ossicles were retrieved, fixed, scanned by $\mu \mathrm{CT}$ for morphometric analysis, and examined histologically by $\mathrm{H} \& \mathrm{E}$ staining. Transplanted $W T$ cells developed a typical ectopic bone/marrow organ (Fig. 3c, left) composed of donor-derived bone, marrow stroma and hostderived hematopoietic cells (asterisk, Fig. 3c, left). The same number of transplanted Sfrp2 KO BMSCs failed to fully resorb the gelatin scaffold material (arrowhead in Fig. 3c, right), and did not completely establish a normal ossicle. $\mu C T$ analysis (Fig. 3d, e) showed less trabecular bone in Sfrp2 KO transplants (Tb.BV/TV), with fewer, thinner and more widely spaced trabeculae (Tb.N, Tb. Th, and Tb.Sp, respectively), that was also structurally altered (structure model index, SMI) (Fig. 3e).

\section{Sfrp2 KO mice display reduced intramembranous bone} regeneration in response to injury

Based on the finding of decreased bone formation by the Sfrp2 KO BMSCs in the in vivo ectopic ossicle assay, we sought to determine if a similar intrinsic defect could be observed in a minor in vivo injury. For this purpose, a drilled hole defect was created in the left femur of Sfrp2 KO and WT littermate mice to assess their intramembranous cortical bone regeneration (Fig. 4a, method modified from Monfoulet et al. $^{33}$ ) keeping in mind that there was no periosteum overlying the defect (therefore, no cartilaginous callus is formed). Fifteen days after surgery, the cortical bone defect and underlying marrow was filled with trabecular bone in $W T$ mice (Fig. 4b, left), but next to no new bone was visible in Sfrp2 KO mice defects (Fig. 4b, right). In addition, a regenerated periosteum was greatly thickened in the $W T$ defect, but not in the Sfrp2 KO defect (Fig. 4b, large black arrows). Indeed, by $\mu C T$ analysis of the regeneration site (Fig. 4C), we observed a three- to five-fold reduction in bone volume (BV/TV), in trabecular number (Tb.N) and trabecular connectivity density (Tb.Conn.D) in Sfrp2 KO compared with their WT littermates, while the trabecular spacing (Tb.Sp) was almost twice as high (Fig. 4d).

\section{Sfrp2 deficiency reduces BMSC/SSC osteogenic differentiation} in vitro

In order to determine the reason for the lack of bone formation in both in vivo models, we next assessed Sfrp2 KO and WT BMSCs for their osteogenic capacity in vitro. At Passage 3 or less, cultures were depleted of hematopoietic CD45 $5^{+}$and $C D 11 b^{+}$cells and induced towards osteogenesis using medium containing dexamethasone/ $\beta$-glycerophosphate/ascorbic acid/ (D/P/A). Three days after induction, Sfrp2 KO cultures showed a significantly decreased expression of the early osteogenic transcription factors, Runx2 and Osterix (Fig. 5a). Addition of recombinant murine Sfrp2 (rm-Sfrp2) restored the expression levels of Runx2 and Osterix (Fig. 5a). Three weeks 
a

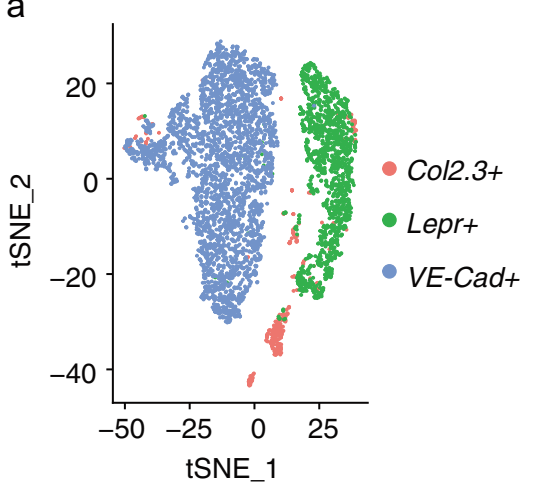

b

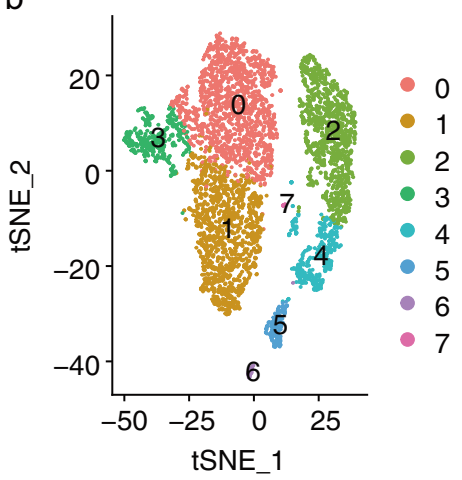

C
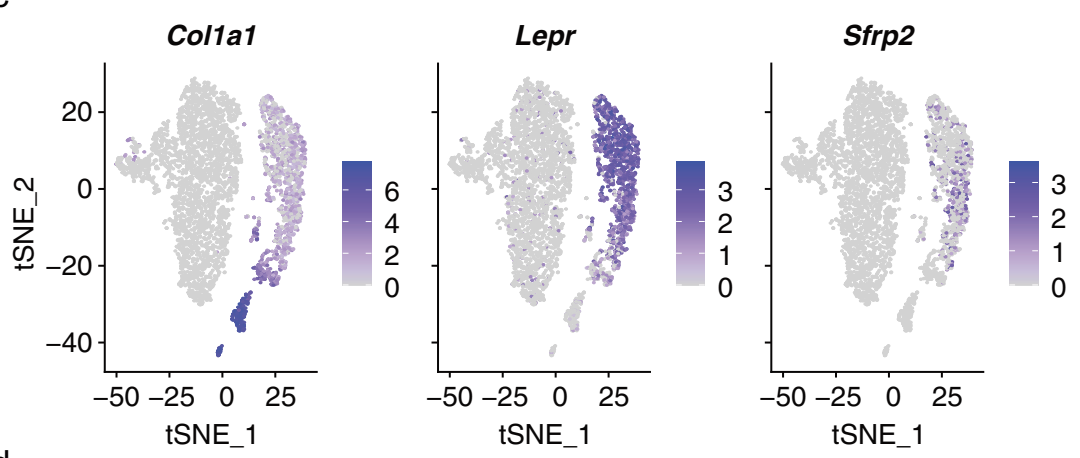

d
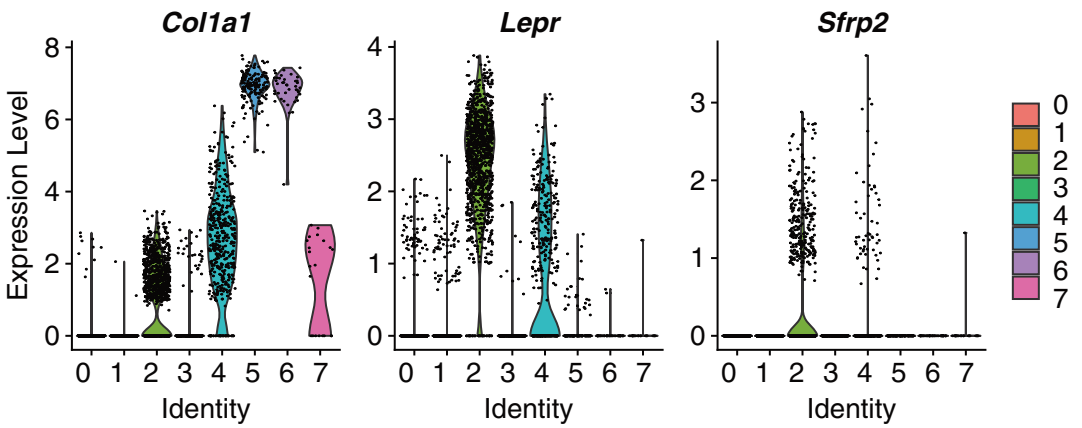

Fig. 1 Analysis of scRNA-seq data from previously published datasets ${ }^{29}$ that assessed non-hematopoietic cell lineages of mouse bone marrow in steady state conditions. a tSNE plot of cre-induced osteoblasts (Col2.3 ${ }^{+}$; red), mesenchymal stromal cells (Lepr ${ }^{+}$; green), and vascular cells (VE-Cad +; blue) and (b) associated clusters. c, d Sfrp2-expressing cells were found to be significantly enriched in cluster 2, a cluster containing the Lepr $^{+}$skeletal stem/progenitors (termed "mesenchymal stromal" cell population by the authors) that also exhibited the greatest number of cells with a high magnitude of Lepr expression

after induction, Sfrp2 KO cultures showed a lower level of calcium accumulation as measured by alizarin red staining, dissolution and spectroscopic measurement (Fig. 5b).

These observations were replicated with an independent assay using an immortalized murine BMSC cell line, DM-5, that maintains the ability to form a complete ectopic ossicle upon in vivo transplantation. ${ }^{34,35}$ Like Sfrp2 KO cells, DM-5 cells silenced for Sfrp2 expression (Fig. 5c-f, Supplementary Fig. 3), showed a significant reduction in the expression of Runx2 and Osterix 3 days after initiation of osteogenic differentiation in vitro using either $10 \mathrm{nmol} \cdot \mathrm{L}^{-1}$ dexamethasone (Fig. $5 \mathrm{c}$ ), or $100 \mathrm{ng} \cdot \mu \mathrm{L}^{-1} \mathrm{BMP}-2$ (Fig. 5e), in addition to $100 \mu \mathrm{mol} \cdot \mathrm{L}^{-1}$ ascorbic acid 2-phosphate and $1 \mathrm{mmol} \cdot \mathrm{L}^{-1} \beta$-glycerol phosphate. Silenced cells in both conditions showed a decreased calcium accumulation at 7 days as well (Fig. 5d, f).

Sfrp2 deficiency reduces Wht signaling in BMSCs/SSCs

Wnt signaling is known to positively regulate osteogenesis, and it has been previously suggested that Sfrp2 is a Wnt antagonist. On the contrary, our data suggested that Sfrp2 is required for normal osteogenesis, both in vivo and in vitro. Consequently, we examined the Wht signaling pathway in WT and Sfrp2 KO BMSCs/SSCs depleted of hematopoietic cells to determine if Sfrp2 is a positive regulator of immature skeletal stem and progenitor cells. We demonstrated that, rather than increased, phosphorylation of the Wnt co-receptor, Lrp6, was reduced in Sfrp2 KO cells (Fig. 6a). It is possible that the reported levels of pLrp6 (Ser1490) also include pLrp5 (ser1493), as per the manufacturer's description. The antibody used is expected to also detect this highly similar epitope. Furthermore, the expression of Wnt downstream target genes Axin2, C-myc, and Cyclin D1, were also reduced in Sfrp2 KO cells, although the reduction in Axin2 was not statistically significant (Fig. 6b). Addition of rm-Sfrp2 showed a non-significant trend toward a rescue in the level of pLrp6 after $5 \mathrm{~h}$ of treatment (Fig. 6a), and increased Axin2 after $24 \mathrm{~h}$, but did not restore C-myc or Cyclin D1 (Fig. 6b).

Similar results were obtained when we replicated the assays in an independent system using immortalized DM-5 BMSCs/SSCs that were treated with Sfrp2-siRNA. pLrp6 was reduced in silenced cells (Fig. 6c), as was Axin2, C-myc, and Cyclin D1 (Fig. 6d). 

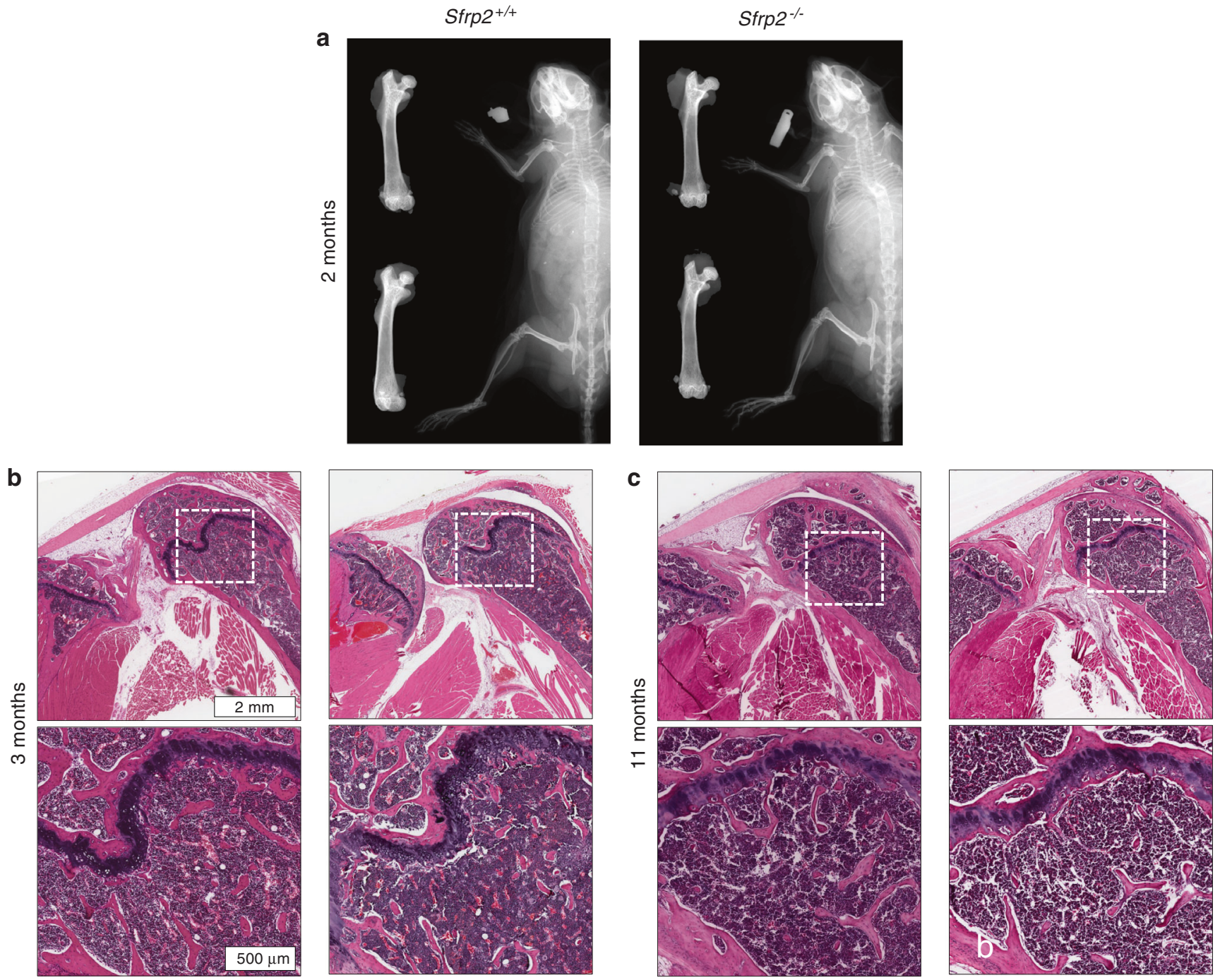

Fig. 2 Sfrp2 KO mice do not exhibit radiological or histological skeletal differences compared with WT mice. a Representative X-ray images of 2-month-old male mice femora and full skeletons. b, c Micrographs (top row) of mid-sagittal H\&E-stained sections of the knee joint, with femora on the right and tibiae on the left, and high magnification micrographs of the sub-metaphyseal area of femora (bottom row), of male mice at 3 months (b), and 11 months (c)

Treatment of the silenced cells with rm-Sfrp2 for $5 \mathrm{~h}$ showed a trend to increase pLrp6, and at $24 \mathrm{~h}$, restored Axin2 expression, with a very modest increase in C-myc, but no increase in Cyclin D1 expression (Fig. 6c, d).

\section{DISCUSSION}

Adult BMSCs and their bmSSC subset have a high therapeutic potential for regenerating new bone, reflected by an abundance of preclinical data. However, many of their theoretical applications in tissue engineering and reconstructive surgery, whereby the cells themselves regenerate lost skeletal elements, remain problematic. This is due, in part, to the difficulty in manufacturing cell products with sufficient numbers of cells with consistent biological activity required for these applications. Extensive ex vivo expansion of BMSCs/SSCs decreases their differentiation capacity in vivo, as the relative abundance of SSCs within BMSC populations progressively decrease with increasing passage number, ${ }^{36}$ (Kuznetsov and Robey, unpublished results). More recently, we showed that even in clinical-grade products, BMSC/SSC differentiation capacity varied greatly depending on the manufacturing method utilized. ${ }^{37}$ In order to develop better in vitro expansion methods able to produce a high yield of multipotent cultured BMSCs/SSCs, it is essential to gain insight into the regulation of SSC self-renewal and to identify biological markers that predict the differentiation potential of cultured BMSCs/SSCs. In a previous study using in vivo ectopic bone formation assays, we demonstrated that: (1) only a small proportion $(12.5 \%)$ of bone marrow CFU-F-derived cell strains are truly multipotent (M-SCDSs), and can differentiate not only into osteoblasts/osteocytes but also into hematopoiesis-supporting stroma and marrow adipocytes; ${ }^{11,38}$ and (2) these cells consistently exhibited a high expression of SFRP2. ${ }^{11}$ This not only supports the use of this WNT modulator as a marker of BMSC multipotency, but also as a potential regulator of bmSSC self-renewal.

Supporting the latter hypothesis, using publicly available datasets of murine bone marrow single-cell RNA sequencing, performed by Tikhonova et al. we observed that Sfrp2 is coexpressed in $\mathrm{Lepr}^{+}$cells, a well-known marker of adult SSCs in mice $^{29}$ (Fig. 1). Moreover, we found that bone marrow from Sfrp2 KO mice had fewer CFU-Fs than their WT littermates (Fig. 3a). However, these mice were only reported to have a minor cartilage defect during fetal development, leading to a subtle brachydactyly phenotype. ${ }^{25}$ Since this study did not include a detailed phenotyping of the adult skeleton of Sfrp2 KO mice, 
a
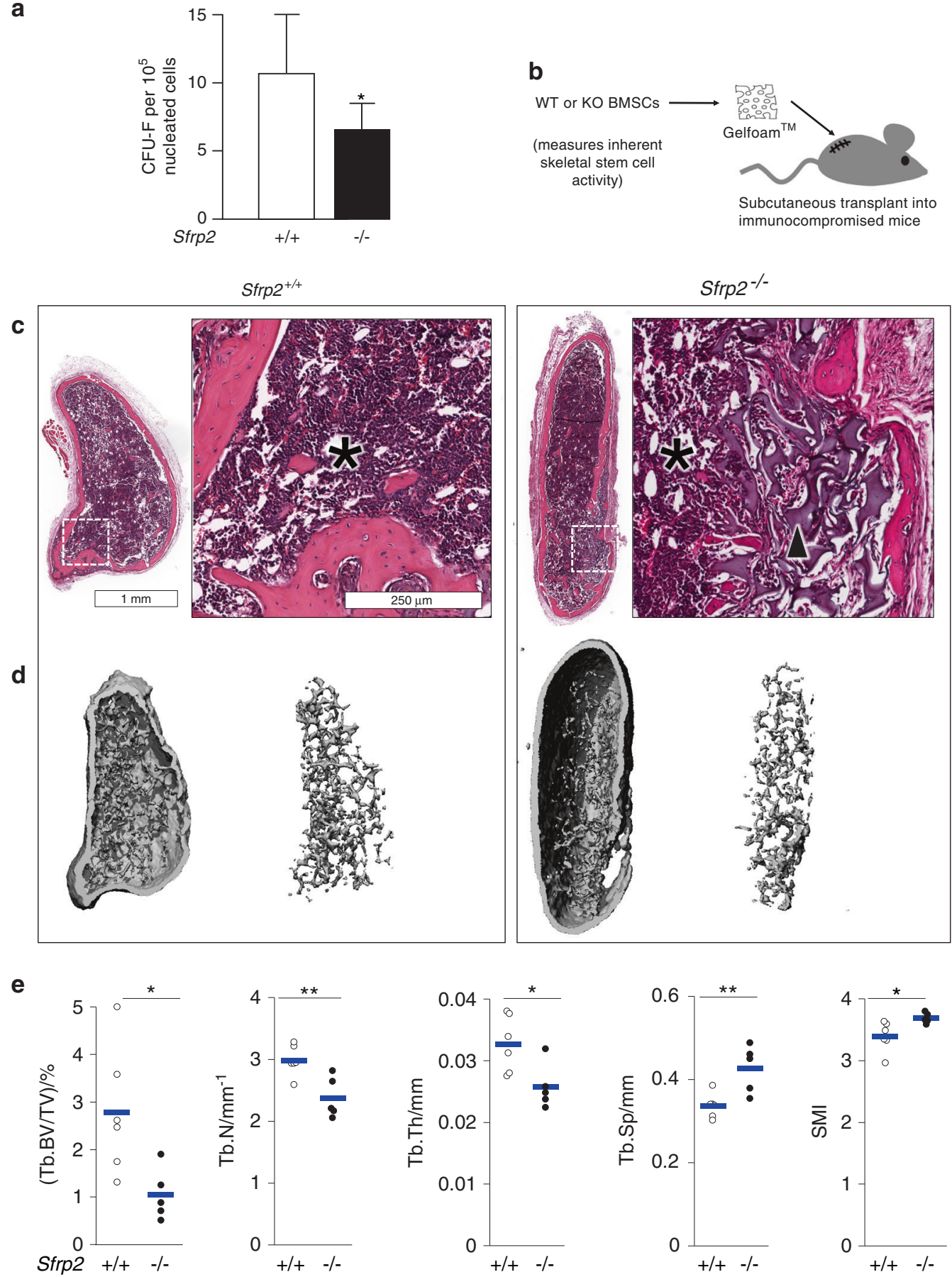

Fig. 3 Sfrp2-deficient BMSCs/SSCs have lower colony-forming efficiency and reduced osteogenic differentiation in ectopic bone transplants. a Colony-forming efficiency assay showing colony-forming units-fibroblasts (CFU-F) per 100000 nucleated bone marrow cells from WT and Sfrp2 KO littermates. b Gelatin sponges were loaded with $2 \times 10^{6}$ BMSCs/SSCs depleted of hematopoietic cells and transplanted into subcutaneous pockets in immunocompromised mice for 8 weeks. c H\&E-stained sections of the transplants. WT BMSCs/SSCs formed a complete bone/marrow organ, with cortical and trabecular bone, stroma that supports hematopoiesis of mouse origin (black asterisk). Sfrp2 $\mathrm{KO}$ BMSCs/SSCs were less osteogenic; transplants showed residual gelatin sponge scaffold material (black arrowheads, far right panel). d $\mu \mathrm{CT}$ reconstructions of transplants and their trabecular compartments. e $\mu C T$ analysis of trabecular bone. Compared with WT transplants, Sfrp2 KO transplants had fewer, thinner trabeculae that were more separated and had abnormal geometry (SMI). (Tb.BV/TV)/\% Trabecular bone volume/Total volume (\%), Tb.Th Trabecular Thickness, Tb.Sp Trabecular Separation, Tb.N Trabecular Number, SMI Structure Model Index. Individual values are shown as five white (WT) or six black circles (Sfrp2 KO), and blue bars represent the mean. ${ }^{*} P<0.05$; ${ }^{* *} P<0.01$

we thoroughly studied their skeleton using X-Rays, DEXA (not shown), $\mu C T$ and histology. Our analyses failed to reveal differences between either young (2-3-month-old) or old (11month-old) sex-matched Sfrp2 KO and WT littermates (Fig. 2, Supplementary Figs. 1, 2), demonstrating that Sfrp2 is not essential for fetal or postnatal modeling and remodeling of the skeleton in mice. Interestingly, Sfrp1 KO mice are also born normal, but Sfrp1 and Sfrp2 double deficiency causes lethality, shortening of the thoracic region and abnormal limb morphogenesis. ${ }^{39}$ This indicates a high degree of redundancy in the 


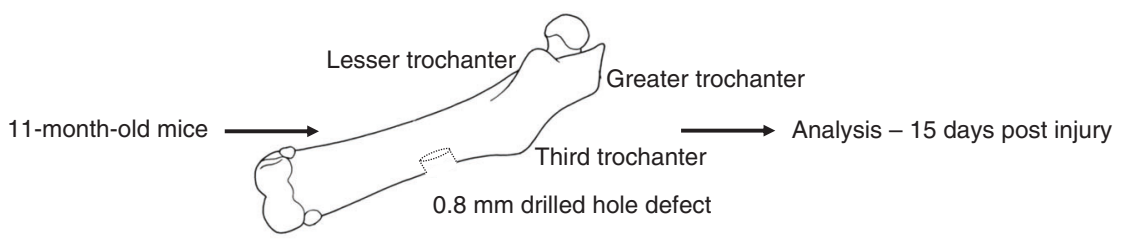

b
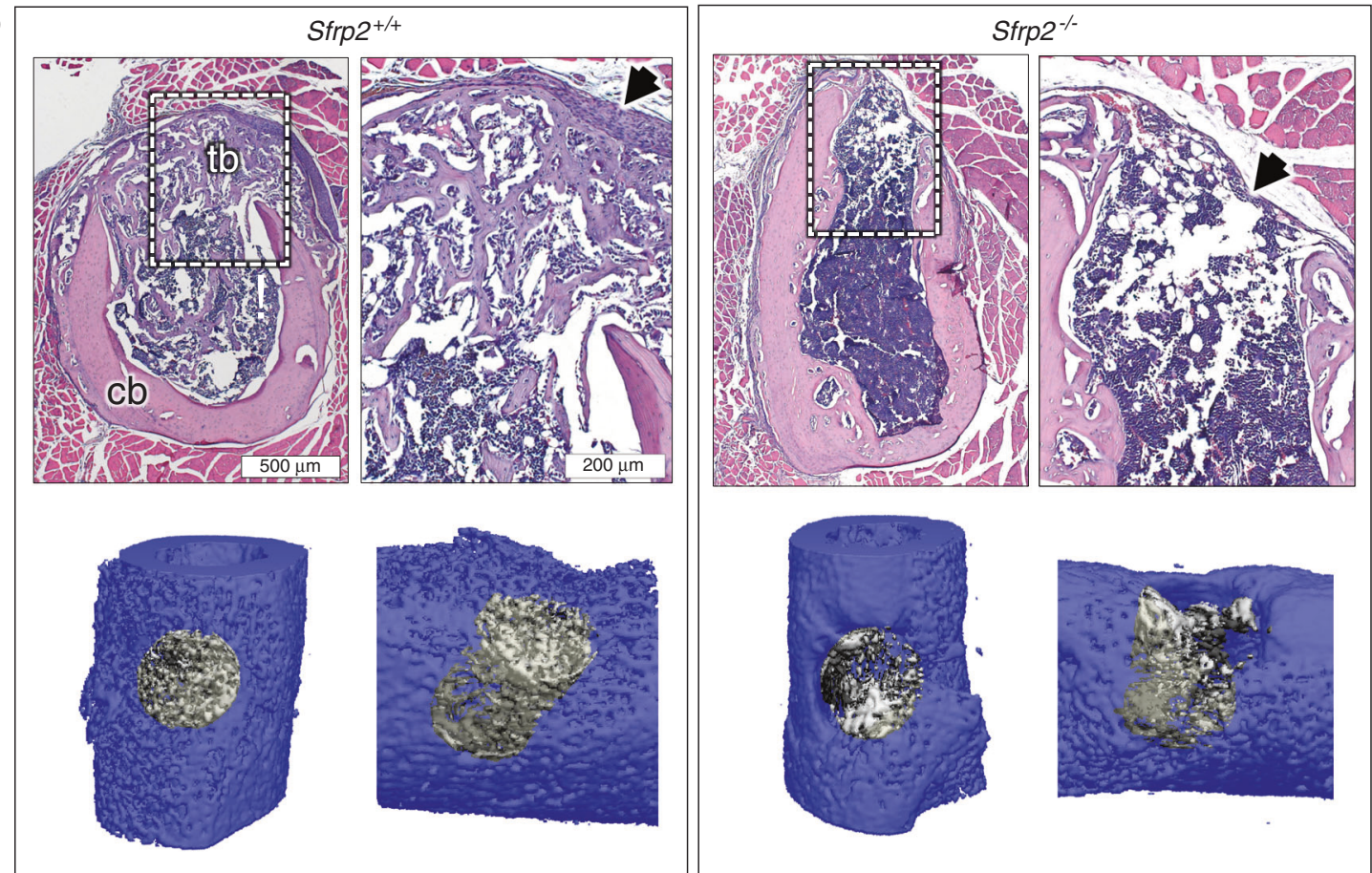

\section{C}
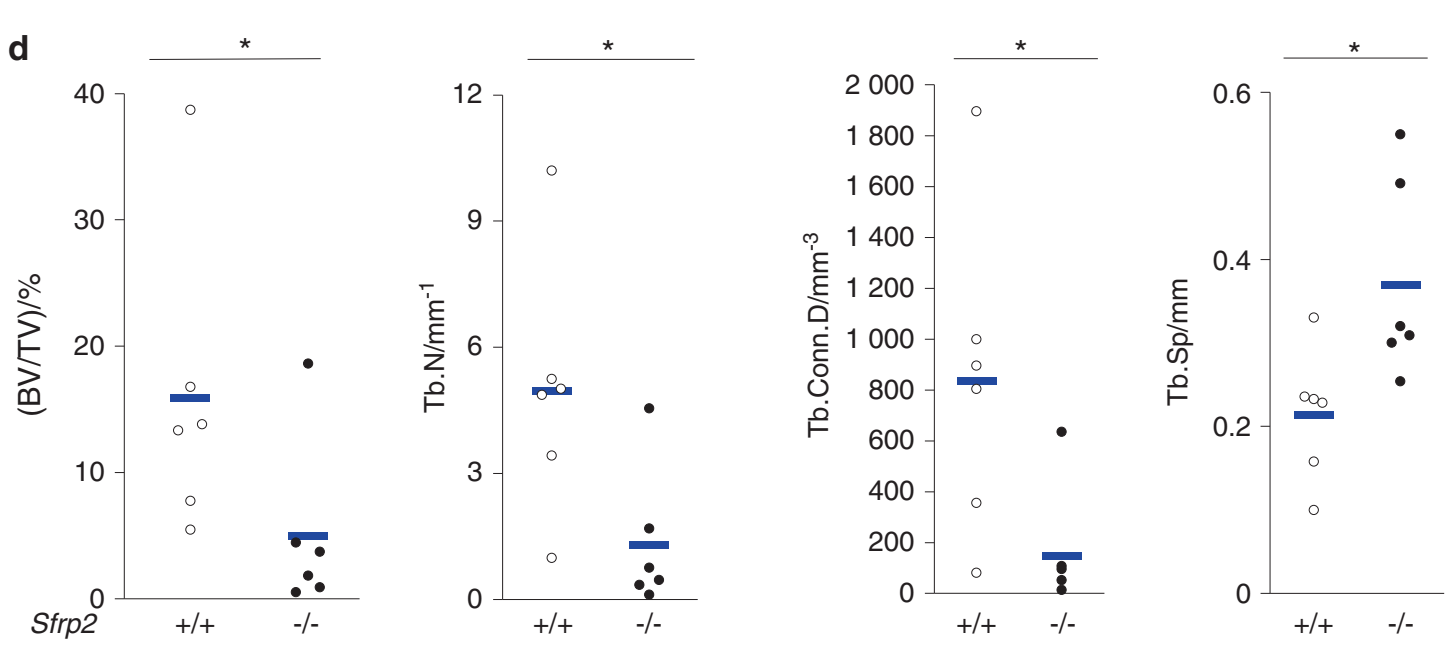

Fig. 4 Sfrp2 KO mice exhibit a bone regeneration defect in vivo. a Drilled bone defect assay. $\mathbf{b}$ Mid-transverse sections of the bone defect stained with H\&E. Periosteal regeneration was evident in WT mice, while Sfrp2 KO mice exhibited little periosteal response (black arrows). Tb trabecular bone, $\mathrm{Cb}$ cortical bone. $\mathrm{c} \mu \mathrm{CT}$ reconstructions showing in gray the drilled volume evaluated. $\mathbf{d}$ Compared with $W T$, the defects in Sfrp2 KO mice had less bone, fewer trabeculae with less connectivity that were more widely spaced. (Tb.BV/TV)/\% Trabecular bone volume/ Total volume (\%), Tb.N Trabecular number, Tb.Conn.D Trabecular connectivity density, Tb.Sp Trabecular separation. Individual values are shown as six white (WT) or five black dots (Sfrp2 KO), and blue bars represent the mean. ${ }^{*} P<0.05$ vs. WT

function of Sfrp1 and 2 during fetal development. Of note, we observed a strong up-regulation of Sfrp1 and to a lesser degree, of Sfrp4, in cultured Sfrp2-KO BMSCs (Supplementary Fig. 5).

SFRPs were initially described as negative WNT modulators. ${ }^{40}$ However, their role in WNT signaling appears to be complex and incompletely understood. SFRPs have been shown to act as antagonists by sequestering WNT via their NTRs and/or CRDs [reviewed in, ${ }^{41,42}$ ] or by direct binding to Frizzled receptors through their CRDs, ${ }^{43}$ However, SFRPs are also suggested to be agonizts by stabilizing extracellular WNT and WNT-Frizzled complexes, ${ }^{44}$ or by direct activation of Frizzled, ${ }^{45}$ or enhancement of ligand-induced signaling. ${ }^{46}$ Different SFRPs may bind to each other, leaving WNT free to promote signaling [proposed in. ${ }^{42}$ ] More recently, SFRPs have been reported to act intracellularly by directly binding to $\beta$-catenin to repress or 
BMSCs

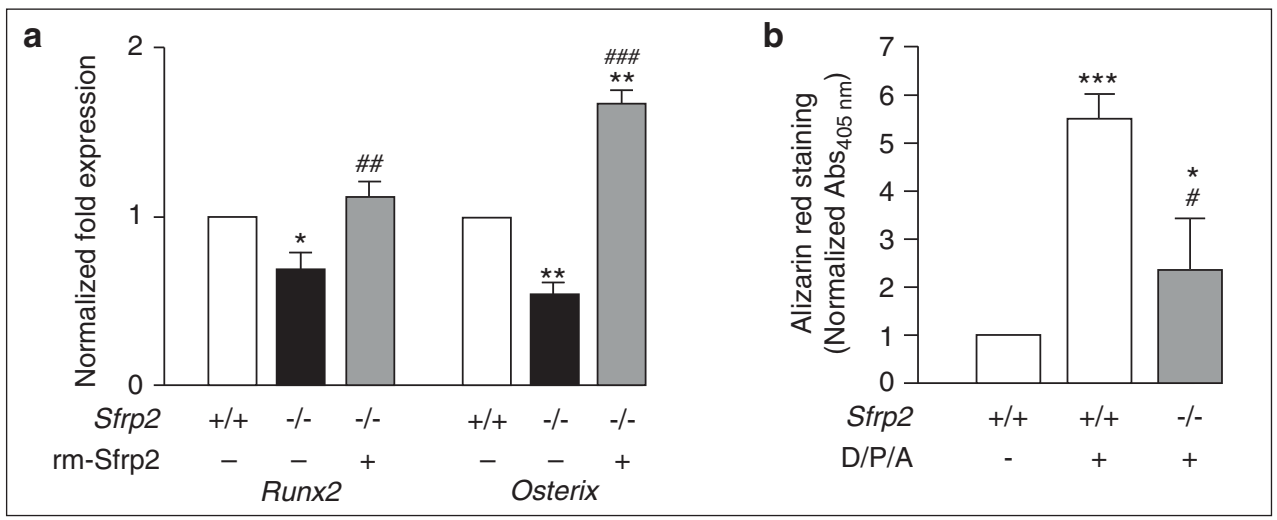

DM-5 immortalized BMSCs

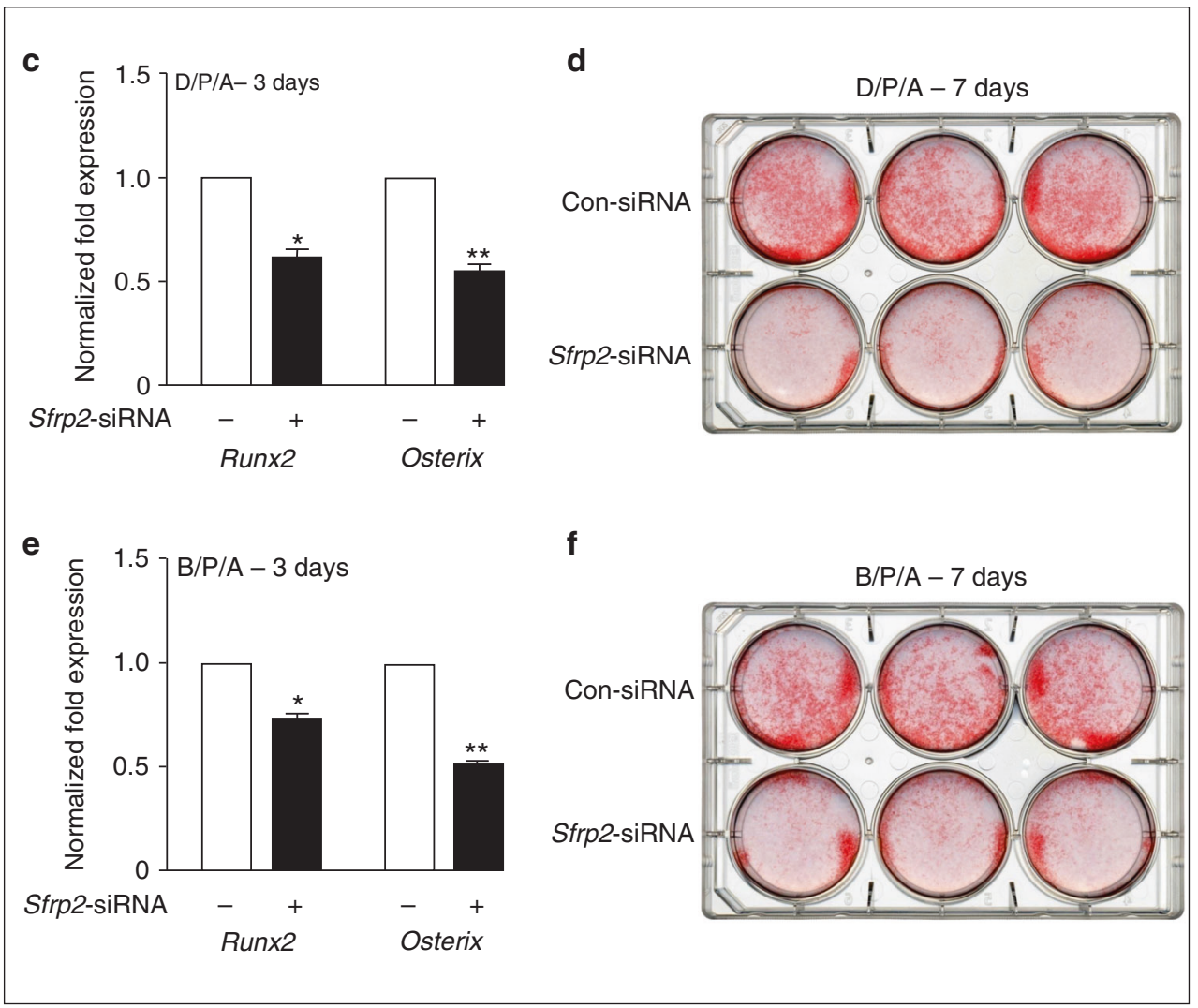

Fig. 5 Sfrp2 deficiency leads to a reduced osteogenic differentiation capacity of BMSCs/SSCs in vitro. a After 3 days of osteogenic differentiation with dexamethasone, $\beta$-glycerol phosphate and L-ascorbic acid 2-phosphate (D/P/A), Sfrp2 KO BMSCs/SSCs showed less expression of Runx2 and Osterix (vs. Gapdh) than WT BMSCs/SSCs. Addition of exogenous rm-Sfrp2 $\left(1 \mu \mathrm{g} \cdot \mathrm{mL}^{-1}\right) \mathrm{normalized} \mathrm{Runx2}$ expression, and increased Osterix expression above WT levels. b After 21 days of osteogenic differentiation (D/P/A), Sfrp2 KO BMSC/SSC cultures were less mineralized than WT cultures, as measured by alizarin red S staining and absorptiometry at $405 \mathrm{~nm}$. ${ }^{*} P<0.05 ;{ }^{* *} P<0.01 ;{ }^{* * *} P<0.01$ vs. WT BMSCs/SSCs; ${ }^{\#} P<0.05 ;{ }^{\# \#} P<0.01 ;{ }^{\# \# \# ~} P<0.01$ vs. Sfrp2 KO BMSCs/SSCs c-f DM-5, an immortalized BMSC/SSC line that retains the ability to form an ectopic ossicle upon transplantation, was transfected with scrambled (Con-siRNA) or Sfrp2-siRNA. After 3 days of osteogenic differentiation with either D/P/A in (c), or BMP-2, $\beta$-glycerol phosphate and L-ascorbic acid 2-phosphate (B/P/A) in (e), cells silenced for Sfrp2 expression showed decreased expression of Runx2 and Osterix (vs. Gapdh). After 7 days of osteogenic differentiation with D/P/A or B/P/A, cells silenced for Sfrp2 expression showed decreased calcium accumulation as shown in alizarin red $S$ stains in (d, f). Data represent mean \pm SD. ${ }^{*} P<0.05 ;{ }^{*} P<$ 0.01 vs. Con-siRNA-treated cells

activate TCF4 recruitment. ${ }^{12}$ Lastly, WNT-independent actions such as regulation of BMP signaling pathways have also been suggested. ${ }^{47,48}$ In light of this complexity, it is not unexpected that the literature reflects diversity in the downstream effects of SFRPs. A current interpretation is that SFRPs' mechanisms of action are highly dynamic, and vary greatly depending on the cell type, differentiation stage and microenvironment. ${ }^{26}$
In our in vitro experiments, Sfrp2 deficiency was associated with a reduced osteogenic differentiation by either dexamethasone/ascorbate/ß-glycerophosphate or BMP-2 osteogenic medium (Fig. 4). Adding rm-Sfrp2 to Sfrp2 KO BMSC/SSC cultures restored the expression of osteogenic markers. This contrasts with previous data showing decreased BMP2-mediated osteogenic differentiation of MC3T3 cells after the addition of exogenous Sfrp2. ${ }^{49}$ However, 


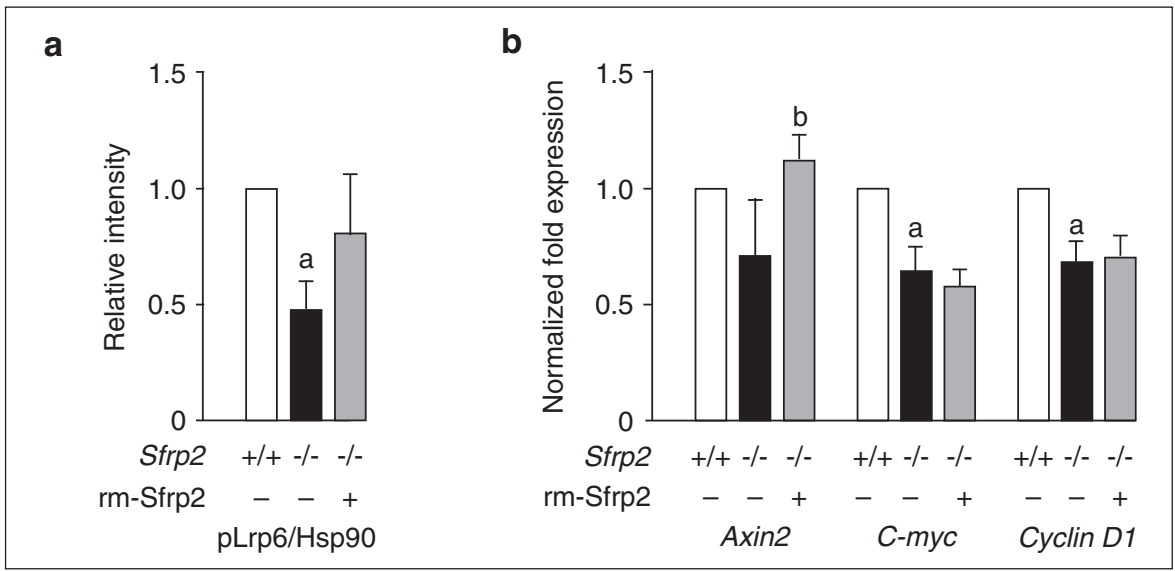

DM-5 immortalized BMSCs

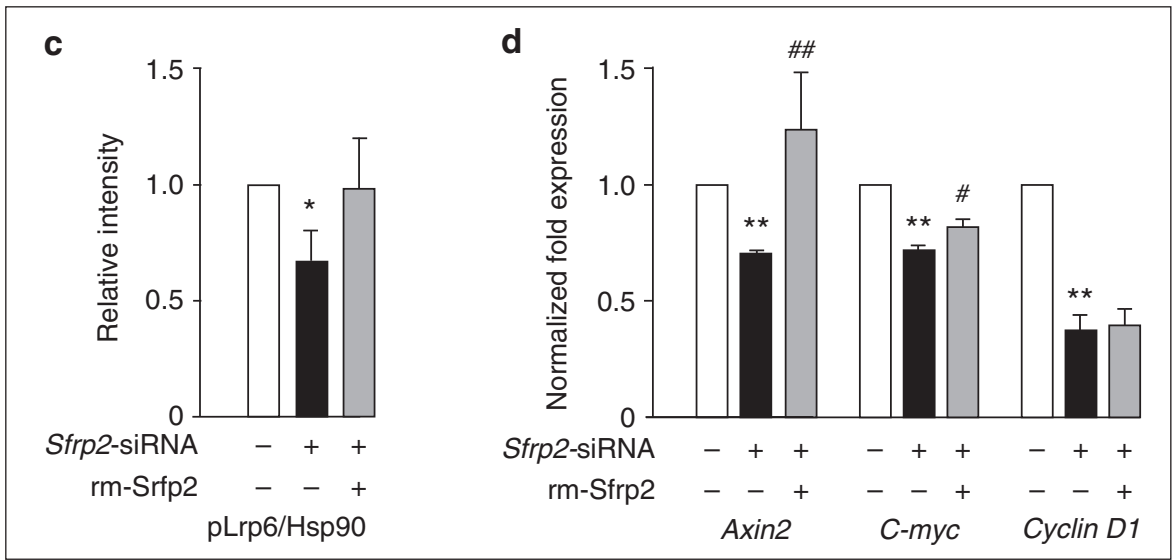

Fig. 6 Sfrp2 deficiency reduces Wnt signaling in murine BMSC/SSCs. a, b BMSCs/SSCs from WT and Sfrp2 KO mice were cultured, depleted of hematopoietic cells and treated with $1 \mu \mathrm{g} \cdot \mathrm{mL}^{-1} \mathrm{rm}$-Sfrp2 or vehicle. C,D DM5 cells were transfected with scrambled (Con-siRNA) or Sfrp2-siRNA and treated with $1 \mu \mathrm{g} \cdot \mathrm{mL}^{-1} \mathrm{rm}$-Sfrp2 or vehicle. a, c Lrp6-Ser1490 phosphorylation, as measured by western blot densitometry versus HSP90, was decreased in Sfrp2-deficient cultures and trended to normalize $5 \mathrm{~h}$ after the addition of rm-Sfrp2. It is possible that the reported levels of pLrp6 (Ser1490) also include pLrp5 (ser1493), as per the manufacturer's description. The antibody used is expected to also detect this highly similar epitope. b, d Expression of Wnt target genes Axin2, C-myc and Cyclin D1 (vs. Gapdh) was decreased in Sfrp2-deficient cultures and partially normalized $24 \mathrm{~h}$ after the addition of $\mathrm{rm}$-Sfrp2. Data are presented as the mean of three or more independent experiments \pm SD. ${ }^{\text {a }} P<$ 0.05 vs. WT BMSCs/SSCs, b $P<0.05$ vs. Sfrp2 KO BMSCs/SSCs. ${ }^{*} P<0.05 ;{ }^{* *} P<0.01$ vs. Con-siRNA treated cells; ${ }^{\#} P<0.05$, ${ }^{\# \#} P<0.01$ vs. Sfrp2-siRNA treated cells

Boland et al. showed that SFRP2 is upregulated during osteogenic differentiation of human $\mathrm{BMSCs}^{50}$ supporting the notion of a complex, differentiation stage-dependent action of SFRP2.

In order to determine if the decrease in osteogenic differentiation in the face of Sfrp2 deficiency was due to a decrease in Wnt signaling, we used two different experimental approaches: (1) BMSCs/SSCs derived from Sfrp2 KO and WT littermates, and (2) knocking down Sfrp2 mRNA expression in immortalized DM-5 cells in vitro (Supplementary Fig. 4). In both cases, abrogation of Sfrp2 expression in undifferentiated osteoprogenitors caused a decrease in phosphorylation of the Wnt co-receptor, Lrp6, and downregulation of Wnt target genes Axin2, C-myc, and Cyclin D1 in vitro, suggesting that Sfrp2 activates Wnt canonical pathway. Addition of rm-Sfrp2 partially restored pLrp6 and completely restored Axin2, but did not restore C-myc or Cyclin D1, possibly due to the $24 \mathrm{~h}$ timepoint used to assess the effects of rm-Sfrp2 restoration on gene expression (Fig. 6).

Our observations of decreased osteogenesis and Wnt activation in Sfrp2-deficient BMSCs/SSCs in vitro can be the result of an overall activating role of Sfrp2 in Wnt modulation in our models, but it can also reflect that the increased levels of Sfrp1 and Sfrp4 observed in Sfrp2 KO BMSCs/SSCs (3-fold and 1.2-fold respectively,
Supplementary Fig. 4) caused increased inhibition of Wnt pathway and osteogenesis. Yet, these two explanations are not mutually exclusive. Both Sfrp1 and Sfrp2 have been reported as Wnt inhibitors. ${ }^{51,52}$ These data suggest that while Sfrp1 and Sfrp2 have many overlapping effects, Sfrp2 has activities that are unique with regard to Wnt signaling. In any event, these possibilities are in agreement with the increasing notion that SFRPs and other WNT modulators are a part of a highly dynamic system that behaves differently depending on the model system used, and is very much influenced by the cellular context and differentiation stage. This highlights the need for in vivo assessment of Sfrp2 in the function of skeletal stem cells and osteoprogenitors. With this aim in mind, we carried out two in vivo assays to study different aspects of the role of Sfrp2 in BMSCs/SSCs.

Producing ectopic bone/marrow organoids with BMSCs/SSCs allows for the specific measurement of BMSC/SSC multipotency. The ability of Sfrp2 KO and WT BMSCs to differentiate into a complete ectopic bone/marrow organ was assessed independently of the systemic effects of Sfrp2 deficiency, as Sfrp2 KO and WT cells were transplanted into an independent organism (a WT host mouse). Sfrp2-deficient BMSCs/SSCs showed a dramatic reduction in their ability to generate a bone de novo, as evidenced 
by the presence of un-resorbed gelatin scaffold detected by histology, and abnormal $\mu C T$ measurements. Interestingly, Sfrp2 deficiency in BMSCs/SSCs did not seem to significantly affect the support of hematopoietic marrow formation in these transplants, which derives from host hematopoietic cells growing on donor stroma (Fig. 3). Consistent with our in vivo ossicle results, Sfrp2 overexpression in stem cells from the apical papilla (SCAPs) exhibited a superior tissue regeneration capacity in a periodontitis/regeneration model in miniature pigs, ${ }^{53}$ and subcutaneous transplantation of Sfrp2 knockdown SCAPs (SCAPs of teeth) demonstrated less dentin-like tissue formation than normal cells. ${ }^{54}$

The drilled mid-diaphyseal femur defect is a relatively minor injury that resolves primarily by intramembranous ossification, with a lower involvement of inflammation, vascularization and resorption than in fracture models driven by endochondral ossification. ${ }^{33}$ Consequently, this assay explores the effects of Sfrp2 deficiency more specifically on the mobilization and osteogenic differentiation of both bmSSCs and periosteal SSCs (pSSCs). Sfrp2 KO mice were virtually unable to repair this minor injury. Sfrp2 deficiency negatively affected the mobilization and differentiation capacity of SSCs. Unlike WT mice, Sfrp2 KO mice exhibited no periosteal thickening (Fig. 4b, black arrows), showing a near-complete absence of periosteal SSC (pSSC) response to the injury. While it is more challenging to directly observe a bmSSC response, there was a complete absence of intramedullary trabeculae in the defect. There was a threefold decrease in de novo bone formation in the drilled area as assessed by $\mu \mathrm{CT}$ analysis (Fig. 4c), along with a very low number of trabeculae present only at the margin of the injury in the Sfrp KO mice. In this model, it is possible that global Sfrp2 deficiency could be affecting SSC-independent processes occurring during bone regeneration, such as vascularization, thereby contributing to the failure to regenerate this minor defect. However, the inability of the Sfrp2 KO mice to heal this minor defect, along with the inability to create a complete bone/marrow organ from their BMSCs/SSCs in the ectopic ossicle model, can be clearly linked to the decrease in CFE of BMSCs/SSCs. This is suggestive of a decrease in self-renewal of the bmSSCs in these mice, and most likely in the self-renewal of the pSSCs, based on the lack of periosteal regeneration. In addition to a decrease in selfrenewal and bone regeneration in vivo, the Sfrp KO BMSC/SSC population also displayed decreased osteogenic differentiation in vitro, due to a decrease in WNT signaling.

Characterizing autocrine/paracrine factors controlling SSCs selfrenewal has important implications for the field of regenerative medicine. Cultured BMSCs/SSCs can potentially repair large segments of bone that are unlikely to adequately heal due to trauma, surgical resection of cancer or skeletal pathologies, but the cell numbers necessary for this goal requires extensive expansion of these cells in vitro. In order to produce enduring regenerated bone, it is essential to guarantee the presence of self-renewing SSCs within BMSC/SSC cultures. ${ }^{55}$ Based on our results, Sfrp2 is dispensable for the development and maintenance of the mouse skeleton in an unchallenged scenario, but appears to be essential for the mobilization and differentiation of bmSSCs and pSSCs into functional osteogenic cells during bone repair and regeneration. Measuring the expression levels of SFRP2 in clinical-grade cultured BMSCs could be useful in predicting their subsequent in vivo proliferation and differentiation in tissue engineering applications. Further studies are needed to unravel the intricacies of the role of Sfrp2 in adult SSC self-renewal and function in response to injury.

\section{MATERIALS AND METHODS}

Determining Sfrp2 expression in previously published scRNA-seq data

To determine the expression of Sfrp2 cells in the mouse nonhematopoietic bone marrow compartment, we analyzed a publicly available single-cell RNA-seq datasets from the GEO series
GSE108892. ${ }^{29}$ These datasets were generated using cells that were isolated from lineage-specific Cre-transgenic mice crossed to a knock-in reporter strain, Rosa26 locus (LoxP-tdTomato). The experimental details for generating these datasets were previously published by Tikhonova et al. ${ }^{29}$ and include cells from the following transgenic mouse crosses: VE-Cad-cre;LoxP-tdTomato (VE-Cad-tdTomato) (GSM2915577) for vascular cells; LEPR-cre; LoxP-tdTomato (LEPR-tdTomato) (GSM2915576) for bone marrow-derived stem/progenitor cells (termed "mesenchymal stromal cells" by the authors); and COL2.3-cre;LoxP- tdTomato (COL2.3-tdTomato; note, COL2.3 refers to the $2.3 \mathrm{~kb}$ of the Col1a1 promotor) (GSM2915575) for osteoblasts.

The datasets were imported into Seurat package version 4.0.1 in $\mathrm{R}^{56}$ and standard workflows were followed with default settings as described in the Seurat online tutorials (https://satijalab.org/ seurat/). We filtered out cells that had less than 200 or more than 6000 genes, and more than 5\% mitochondrial counts using the subset function. The datasets were integrated using the Seurat built-in integration functions. Blood cell lineage clusters were identified based on the expression of Ptprc, Lyz2, or hemoglobin genes, and removed by subsetting. After removal of blood cells, the data was r-eclustered and tSNE plots generated. This process resulted in seven clusters for which differentially expressed genes were calculated.

\section{Sfrp2 KO mice}

Sfrp2 KO mice were kindly provided by Drs. Roy Morello and Brendan Lee at the Baylor College of Medicine, Houston, $\mathrm{TX}^{25}$ and genotyped by touchdown PCR and electrophoresis in $2 \%$ agar using the following primers (derived from NCBI Reference Sequence NC_000069.7): Wildtype (WT) forward 5'-GAGGTGAAAGAGGTTGGTCGT-3', Sfrp2 KO forward 5'-TTGAGCCCGGTGTTACTGGAG-3' and common reverse 5'-AAACCTTATGACCTCCTGTGAGG$3^{\prime}$. The Sfrp2 KO amplicon is approximately $700 \mathrm{nt}$ and the WT amplicon is $800 \mathrm{nt}$. This study was conducted under an institutionally approved protocol in accordance with the $\mathrm{NIH} /$ NIDCR Animal Care and Use guidelines (\#13-687).

X-ray and microcomputed tomography $(\mu \mathrm{CT})$ analyses

To characterize the bone phenotype of Sfrp2 KO mice, whole 8week-old mice and dissected femora were imaged using an MX-20 $\mathrm{X}$-ray device (Faxitron, Tucson, AZ). Samples were exposed for $15 \mathrm{~s}$ at $30 \mathrm{kV}$ using Kodak X-Omat film, which was then electronically scanned. Skulls, femora and ectopic transplants were imaged by microcomputed tomography $(\mu \mathrm{CT}, 70 \mathrm{kVp}, 85 \mu \mathrm{A}, 0.3 \mathrm{~s}$ integration time, $1000 \mu \mathrm{m}^{3}$ voxel resolution, Scanco Medical AG, Brüttisellen, Switzerland). Intact femora (3 months and 11 months, males and females) were analyzed for histomorphometric parameters as previously described. ${ }^{57,58}$

\section{Tissue processing and histology}

Anesthetized mice were euthanized by terminal vascular perfusion and fixation by sequentially injecting $10 \mathrm{~mL}$ of ice-cold PBS and $10 \mathrm{~mL}$ of ice-cold $4 \%$ formaldehyde into the left ventricle of the heart [modified from ref. ${ }^{59}$ ]. Samples were then dissected, post-fixed overnight with $4 \%$ formaldehyde at $4{ }^{\circ} \mathrm{C}$ and stored in PBS with $0.025 \%$ sodium azide (Sigma-Aldrich). Mice used for dissection of calvariae were perfused only with PBS and these samples were used for an osteoclastogenesis (TRAP) assay (see below). Samples used for hematoxylin and eosin (H\&E) staining were decalcified in $250 \mathrm{mmol} \cdot \mathrm{L}^{-1}$ EDTA in phosphate buffer $(\mathrm{pH}$ 7.4) at $4{ }^{\circ} \mathrm{C}$. Decalcification was monitored by weekly $X$ rays until no evidence of calcified tissue was observed. Tissue was then embedded in paraffin, cut into $5 \mu \mathrm{m}$ sections and stained. Microscopic images were obtained with a Leica Aperio Scancope (Leica, Buffalo Grove, IL) or a Zeiss Axioplan 2 microscope 
equipped with an AxioCam HRc camera (Carl Zeiss, Inc., Thornwood, NY).

Tartrate-resistant acid phosphatase activity assay (TRAP)

Calvariae were dissected, rinsed in ice-cold PBS, fixed in acetone citrate for $30 \mathrm{~s}$, washed briefly in PBS and transferred to a prewarmed garnet/tartrate solution from an Acid Phosphatase kit (Sigma-Aldrich). Calvariae were stained at $37^{\circ} \mathrm{C}$, protected from light, and assessed every $5 \mathrm{~min}$. Stained specimens were stored in $70 \%$ ethanol and photographed using a dissection microscope. ${ }^{30,35}$

Colony-forming efficiency assay and cell culture

Eight- to twelve-week-old, age and sex-matched mice were euthanized, and femora and tibiae were aseptically removed and cleaned of soft tissues. Marrow cavities of the bones were flushed into growth medium [a-minimum essential medium (a-MEM), $2 \mathrm{mmol} \cdot \mathrm{L}^{-1}$ glutamine (Invitrogen, Carlsbad, CA), penicillin $\left(100 \mathrm{U} \cdot \mathrm{mL}^{-1}\right)$, streptomycin sulfate $\left(100 \mu \mathrm{g} \cdot \mathrm{mL}^{-1}\right.$; Invitrogen), 2-mercaptoethanol $\left(0.1 \mathrm{~mol} \cdot \mathrm{L}^{-1}\right)$, and $20 \%$ lotselected non-heat-inactivated fetal bovine serum] using a nutrient medium-filled syringe attached to a $23 \mathrm{G}$ needle. To determine CFE, a freshly prepared single-cell suspension of bone marrow cells was plated at low (clonal) density in $25-\mathrm{cm}^{2}$ plastic culture flasks $\left(4 \times 10^{4}\right.$ nucleated cells per $\left.\mathrm{cm}^{2}\right)$ with growth medium and cultured for 10 days at $37^{\circ} \mathrm{C}, 5 \% \mathrm{CO}_{2}$ without changing the medium. Next, flasks were rinsed once with Hanks' Balanced Salt Solution (GIBCO-Life Technologies, Grand Island, NY), fixed with $100 \%$ methanol for $30 \mathrm{~min}$, and stained with an aqueous solution of saturated methyl violet for $30 \mathrm{~min}$. Flasks were rinsed three times with distilled water and dried overnight. Dried, stained colonies ( $>50$ cells) were counted using a dissecting microscope, as described. ${ }^{60}$

To establish non-clonal BMSC cultures, single-cell suspensions of bone marrow $\left(6-8 \times 10^{7}\right.$ nucleated cells) were also plated into $75-\mathrm{cm}^{2}$ flasks in growth medium and cultured for 7 days at $37^{\circ} \mathrm{C}$, $5 \% \mathrm{CO}_{2}$. Adherent cells were then detached by incubating for $30 \mathrm{~min}$ at $37^{\circ} \mathrm{C}$ in collagenase type IV $\left(2 \mathrm{mg} \cdot \mathrm{mL}^{-1}\right.$ in a-MEM, Invitrogen), and then for $5 \mathrm{~min}$ at $37^{\circ} \mathrm{C}$ in trypsin $(0.05 \%$ in $0.53 \mathrm{mmol} \cdot \mathrm{L}^{-1}$ EDTA, Invitrogen). Cultures were maintained for up to 3 weeks (3 passages) with medium changes every 3 days. ${ }^{35,61}$ Cultures were depleted of $\mathrm{CD}_{4} 5^{+}$and $\mathrm{CD} 11 \mathrm{~b}^{+}$cells using a magnetic sorting system following the manufacturer's instructions (MACS, Miltenyi Biotec Inc., Auburn, CA, USA), reseeded, and used 24 h later. $^{62}$

\section{Ectopic bone/marrow organ (ossicle) formation}

$2 \times 10^{6}$ BMSCs/SSCs were suspended in $15 \mu \mathrm{L}$ of standard growth medium and absorbed into $7 \mathrm{~mm} \times 5 \mathrm{~mm} \times 5 \mathrm{~mm}$ collagen sponge cubes (Gelfoam , Pfizer, New York, NY). NSG immunodeficient mice (NOD-scid IL2Rgnull, Jackson Laboratory) were anesthetized with isoflurane $(2 \%-5 \%$ in 02$)$. After shaving the fur, a longitudinal incision of $1 \mathrm{~cm}$ was made on the back of the animals, and up to four transplants were placed subcutaneously into the flanks of the mice. The incision was then closed using surgical clips (Fig. 3b). Full body radiograms where taken every 2 weeks using an IVIS Lumina-XR in vivo imaging system to track transplant calcification. Eight weeks after surgery, mice were euthanized, and transplants were dissected and processed for image analyses. For $\mu \mathrm{CT}$ analyses, a volume of interest (VOI) corresponding to the trabecular content of the transplants was generated using a semi-automatic custom Scanco evaluation software script and analyzed using the script designed for trabecular bone. For the three-dimensional binarized reconstructions, samples were segmented using a relative threshold of 180/1 000, sigma $1 / 5$ and support 2/9 using Scanco's reconstruction software. After scanning, the ossicles were processed for histology as described above.
Drilled hole bone defect

Eleven-month-old mice were anesthetized with isoflurane (2\%-5\% in $\mathrm{O}_{2}$ ). The surgical procedure used was a modification of a model previously described. ${ }^{33}$ Briefly, a $1 \mathrm{~cm}$ incision was placed on the lateral side of the thigh along the femur. The femoral diaphysis was exposed by pulling apart the muscle fibers with surgical forceps. Holes $(0.8 \mathrm{~mm})$ were created by using an electric surgical drill (Ideal Micro Drill, CellPoint Scientific, Gaithersburg, MD) and drilling through the periosteum and cortex at the lateral aspect of the mid-diaphysis. The holes were located immediately distal to the third femoral trochanter, which was used as an anatomical landmark (Fig. 4a). The region was continuously irrigated with sterile PBS to avoid thermal necrosis. Five $\mathrm{mL}$ of PBS was used to gently rinse bone fragments from the holes, with care taken not to dislodge the marrow. Muscles were realigned and incisions were closed with tissue glue (3 M Vetbond ${ }^{\oplus}$, Saint Paul, MN). Animals were $X$-rayed (Lumina-XR, PerkinElmer) immediately after surgery to check the location of the drilled hole. 15 days after surgery, animals were euthanized, and tissue was processed as described below. The femora were scanned by $\mu \mathrm{CT}$ with a VOI selected as a cylindrical region of $800 \mu \mathrm{m}$ in diameter with curved ends, to encompass the original bone defect and following the curvature of both the periosteum and endosteum of the femur. The newly formed bone in the defect was analyzed using a Scanco script designed for trabecular bone. Bones were subsequently processed for histological analysis.

In vitro osteogenic differentiation and alizarin red staining Osteogenic differentiation of BMSCs in vitro was performed in standard growth medium that was supplemented with $10^{-8} \mathrm{~mol} \cdot \mathrm{L}^{-1}$ dexamethasone, $1 \mathrm{mmol} \cdot \mathrm{L}^{-1} \quad \beta$-glycerol phosphate, $100 \mu \mathrm{mol} \cdot \mathrm{L}^{-1}$ I-ascorbic acid 2-phosphate, (D/P/A) (Sigma-Aldrich, St. Louis, MO, USA). Cultures were monitored daily by phase contrast microscopy for evidence of mineralization, and osteogenic medium was changed every 3 days. For alizarin red staining, cultures were fixed with cold $70 \%$ ethanol for $1 \mathrm{~h}$ at room temperature and stained with a $2 \%$ aqueous solution of alizarin red (Sigma-Aldrich). In some cases, the relative level of $\mathrm{Ca}^{2+}$ accumulation was quantified by elution of bound Alizarin Red with $0.5 \mathrm{ml}$ of $5 \%$ SDS in $0.5 \mathrm{~N} \mathrm{HCl}$ for $30 \mathrm{~min}$ at room temperature, and the absorbance was measured at $405 \mathrm{~nm}$. For some experiments, DM-5 cells, a spontaneously immortalized murine BMSC cell line isolated in our laboratory ${ }^{34,35}$ were used. Cells were thawed and cultured as described above. In some experiments, DM-5 cells were cultured in medium in which dexamethasone was replaced with $100 \mathrm{ng} \cdot \mathrm{LL}^{-1}$ of recombinant human BMP2 (B/P/A) (R\&D Systems, Minneapolis MN).

\section{siRNA transfection}

Immortalized DM-5 cells were used to knockdown Sfrp2 expression with siRNA due to the ease in which they transfect and their proven osteogenic differentiation capacity in vitro and in vivo. Cells were plated in standard growth medium without penicillin and streptomycin such that they were between $30 \%$ to $50 \%$ confluent after $24 \mathrm{~h}$. For 1 well of a 24 well dish, 20 pmols of either Sfrp2-siRNA (ID s73481, Invitrogen) or control (scrambled) siRNA (Silencer Select Negative Control \#1, Invitrogen) were combined with $1.25 \mu \mathrm{L}$ of Lipofectamine RNAiMAX (Invitrogen) in Opti-MEM ${ }^{\oplus}$ in Reduced Serum Medium (Invitrogen), and added to the cells. For larger vessels, the amount of siRNA and Lipofectamine used was scaled up proportionally. The medium was not changed until at least $18 \mathrm{~h}$ after transfection.

Treatment with recombinant murine Sfrp2 (rm-Sfrp2) In some experiments, WT and Sfrp2 KO BMSCs/SSCs depleted of $\mathrm{C}_{4} 5^{+}$and CD $11 \mathrm{~b}^{+}$cells, or DM-5 cells silenced for Sfrp2 expression, were treated with recombinant mouse Sfrp2 $\left(\mathrm{rm}\right.$-Sfrp2, $1 \mu \mathrm{g} \cdot \mathrm{mL}^{-1}$, R\&D Systems, Minneapolis, MN) or vehicle (phosphate buffered solution, PBS) added into fresh medium. 
RNA extraction and qRT-PCR

BMSCs/SSCs were harvested 3 days after the addition of osteogenic medium for determination of mRNA expression of Runx2 and Osx, or after $24 \mathrm{~h}$ of treatment with rm-Sfrp2, for determination of mRNA expression of Axin2, C-myc and Cyclin D1. Immortalized DM- 5 cells were harvested 3 days after the addition of osteogenic medium and 4 days after treatment with siRNA. RNA was isolated using RNeasy Mini Kits (Qiagen, Valencia, CA) and Qiashredder columns (Qiagen) according to the manufacturer's protocol. When additional cleanup of the RNA was necessary, RNA Clean Up and Concentrator-5 columns (Zymo Research Labs, Irvine, CA) were used according to the manufacturer's protocol. RNA was reverse transcribed by using SuperScript III First-Strand Synthesis System (Invitrogen). qRT-PCR analysis was carried out using $\mathrm{iQ}^{\mathrm{TM}}$ SYBR Green Supermix (Bio-Rad Laboratories, Hercules, CA) on a Bio-Rad CFX96 ${ }^{\text {TM }}$ Real-Time PCR Detection System (BioRad Laboratories). cDNA quantification was carried out using the comparative cycle threshold method $(\Delta \Delta C T)$ using Gapdh as a housekeeping gene. Primers used for amplification are listed in Supplementary Table 1.

Western blot

Cultures were harvested $5 \mathrm{~h}$ after treatment with $\mathrm{rm}$-Sfrp 2 for protein extraction for determination of pLrp6 levels. Protein was extracted from cell cultures using PhosphoSafe Extraction Reagent (Novagen, Madison, WI) supplemented with $1 \mu \mathrm{g} \cdot \mathrm{mL}^{-1}$ protease inhibitor cocktail (Roche). Protein extracts were centrifuged at $12000 \mathrm{r} \cdot \mathrm{min}^{-1}$ for $20 \mathrm{~min}$ at $4^{\circ} \mathrm{C}$, and equal amounts of lysate subjected to SDS-polyacrylamide gel electrophoresis using $4 \%-12 \%$ Bis-Tris precast polyacrylamide gels (NuPage, Invitrogen) and MOPS buffer (Invitrogen). Protein was then blotted onto polyvinylidene difluoride (PVDF) membranes (Immobilon- $\mathrm{FL}$, Millipore, Darmstadt, Germany). Blots were blocked with Odyssey Blocking Buffer (Li-Cor, Lincoln, NE) and then incubated overnight with anti-pLrp6S1490 (1:1 000; Catalog \#2568; Cell Signaling Technology, Danvers, MA). It is possible that the levels of pLrp6 (Ser1490) reported here also include pLrp5 (ser1493), as per the manufacturer's description. The antibody used is expected to also detect this highly similar epitope. Hsp90 (1:1 000; sc-13119; Santa Cruz Biotechnology, Dallas, TX) labeling was carried out by incubating the blot at room temperature for $1 \mathrm{~h}$. Protein bands were visualized by use of fluorescently labeled secondary antibody (1:10 000; Li-Cor), and scanned and quantified with an Odyssey scanner (Li-Cor). The relative protein expression levels were normalized to Hsp90 expression levels.

Statistical analysis

The number of independent experiments and replicates are indicated in Supplementary Table 2. Data are expressed as mean \pm SEM for all values. Results were evaluated using unpaired Student's $t$ tests, except for the drilled hole defect model, in which the $t$ test was paired (comparing littermates). Analysis was performed using GraphPad Prism 6 software (GraphPad Software, La Jolla, CA). $P<0.05$ was considered significantly different.

\section{ACKNOWLEDGEMENTS}

The authors are grateful to Dr. Anastasia N. Tikhonova and her co-authors for publicly sharing their mouse bone marrow single-cell RNA expression datasets, to Drs. Brendan Lee and Roy Morello at the Baylor College of Medicine for generously providing the Sfrp2 KO mouse model, to Dr. Larry Fisher, NIDCR, for critically reading this manuscript, and to Ms. Li Li for excellent histological services. This research was supported by the Division of Intramural Research, National Institute of Dental and Craniofacial Research, a part of the Intramural Research Program of the National Institutes of Health, Department of Health and Human Services. Open Access funding provided by the National Institutes of Health (NIH).

\section{AUTHOR CONTRIBUTIONS}

L.F.C._luis.fernandezdecastrodiaz@nih.gov-designed and performed experiments, interpreted data, and contributed to writing the paper. B.J.S.-bsworder@stanford. edu—designed and performed experiments, interpreted data. B.M.—byron.mui@nih. gov-performed experiments, contributed to writing the paper. K.F.-katarzyna. futrega@nih.gov-designed and performed experiments, interpreted data, contributed to writing the paper. A.B.—adberendsen@gmail.com—designed and performed experiments, interpreted data. M.P.—matthew.david.phillips@gmail.com—designed and performed experiments, interpreted data. N.J.B.-Nathan.Burbach@gmail.comperformed experiments, interpreted data. N.C.—nrufman@gmail.com-performed experiments, interpreted data. S.A.K.—skuznetsov@dir.nidcr.nih.gov-performed experiments, interpreted data. Y.G.-yankel@tauex.tau.ac.il—designed experiments, interpreted data. K.H._kholmbeck@sund.ku.dk—designed experiments, interpreted data. P.G.R.—pamela.robey@nih.gov—designed experiments, interpreted data, and contributed to writing the paper.

\section{ADDITIONAL INFORMATION}

Supplementary information The online version contains supplementary material available at https://doi.org/10.1038/s41413-021-00169-7.

Competing interests: The authors declare no competing interests.

\section{REFERENCES}

1. Owen, M. \& Friedenstein, A. J. Stromal stem cells: marrow-derived osteogenic precursors. Ciba Found. Symp. 136, 42-60 (1988).

2. Bianco, P., Robey, P. G. \& Simmons, P. J. Mesenchymal stem cells: revisiting history, concepts, and assays. Cell Stem Cell 2, 313-319 (2008).

3. Bianco, P. \& Robey, P. G. in Handbook of Adult and Fetal Stem Cells. (ed Lanza, R. P.) 415-424 (Academic Press, 2004).

4. Chan, C. K. et al. Identification and specification of the mouse skeletal stem cell. Cell 160, 285-298 (2015).

5. Chan, C. K. F. et al. Identification of the Human Skeletal Stem Cell. Cell 175, 43-56 e21 (2018).

6. Mizuhashi, K. et al. Resting zone of the growth plate houses a unique class of skeletal stem cells. Nature 563, 254-258 (2018).

7. Duchamp de Lageneste, $O$. et al. Periosteum contains skeletal stem cells with high bone regenerative potential controlled by Periostin. Nat. Commun. 9, 773 (2018).

8. Debnath, S. et al. Discovery of a periosteal stem cell mediating intramembranous bone formation. Nature 562, 133-139 (2018).

9. Ono, N., Balani, D. H. \& Kronenberg, H. M. Stem and progenitor cells in skeletal development. Curr. Top. Dev. Biol. 133, 1-24 (2019).

10. Ambrosi, T. H., Longaker, M. T. \& Chan, C. K. F. A revised perspective of skeletal stem cell biology. Front. Cell Dev. Biol. 7, 189 (2019).

11. Sworder, B. J. et al. Molecular profile of clonal strains of human skeletal stem/ progenitor cells with different potencies. Stem Cell Res. 14, 297-306 (2015).

12. Liang, C. J. et al. SFRPs are biphasic modulators of wnt-signaling-elicited cancer stem cell properties beyond extracellular control. Cell Rep. 28, 1511-1525.e1515 (2019).

13. Baron, R. \& Kneissel, M. WNT signaling in bone homeostasis and disease: from human mutations to treatments. Nat. Med. 19, 179-192 (2013).

14. Canalis, E. Wnt signalling in osteoporosis: mechanisms and novel therapeutic approaches. Nat. Rev. Endocrinol. 9, 575-583 (2013).

15. Schupbach, D., Comeau-Gauthier, M., Harvey, E. \& Merle, G. Wnt modulation in bone healing. Bone 138, 115491 (2020).

16. Bennett, C. N. et al. Regulation of osteoblastogenesis and bone mass by Wnt10b. Proc. Natl. Acad. Sci. U.S.A. 102, 3324-3329 (2005).

17. Gong, Y. et al. LDL receptor-related protein 5 (LRP5) affects bone accrual and eye development. Cell 107, 513-523 (2001).

18. Boyden, L. M. et al. High bone density due to a mutation in LDL-receptor-related protein 5. N. Engl. J. Med. 346, 1513-1521 (2002).

19. Little, R. D. et al. A mutation in the LDL receptor-related protein 5 gene results in the autosomal dominant high-bone-mass trait. Am. J. Hum. Genet. 70, 11-19 (2002).

20. Babij, P. et al. High bone mass in mice expressing a mutant LRP5 gene. J. Bone Min. Res. 18, 960-974 (2003).

21. Minear, S. et al. Wnt proteins promote bone regeneration. Sci. Transl. Med. 2 $29 r a 30$ (2010).

22. Kim, J. B. et al. Bone regeneration is regulated by wnt signaling. J. Bone Min. Res. 22, 1913-1923 (2007).

23. Hassan, M. Q. et al. miR-218 directs a Wnt signaling circuit to promote differentiation of osteoblasts and osteomimicry of metastatic cancer cells. J. Biol. Chem. 287, 42084-42092 (2012). 
24. Sathi, G. A. et al. Secreted frizzled related protein (sFRP)-2 inhibits bone formation and promotes cell proliferation in ameloblastoma. Oral. Oncol. 45, 856-860 (2009).

25. Morello, R. et al. Brachy-syndactyly caused by loss of Sfrp2 function. J. Cell Physiol. 217, 127-137 (2008)

26. Xavier, C. P. et al. Secreted Frizzled-related protein potentiation versus inhibition of Wnt3a/beta-catenin signaling. Cell. Signal. 26, 94-101 (2014).

27. Hua, Y. et al. Oligomerization of Frizzled and LRP5/6 protein initiates intracellular signaling for the canonical WNT/beta-catenin pathway. J. Biol. Chem. 293, 19710-19724 (2018).

28. Cho, S. W. et al. Differential effects of secreted frizzled-related proteins (sFRPs) on osteoblastic differentiation of mouse mesenchymal cells and apoptosis of osteoblasts. Biochem. Biophys. Res. Commun. 367, 399-405 (2008).

29. Tikhonova, A. N. et al. The bone marrow microenvironment at single-cell resolution. Nature 569, 222-228 (2019).

30. Holmbeck, K. et al. MT1-MMP-deficient mice develop dwarfism, osteopenia, arthritis, and connective tissue disease due to inadequate collagen turnover. Cell 99, 81-92 (1999).

31. Kuznetsov, S. A., Mankani, M. H., Bianco, P. \& Robey, P. G. Enumeration of the colony-forming units-fibroblast from mouse and human bone marrow in normal and pathological conditions. Stem Cell Res. 2, 83-94 (2009).

32. Krebsbach, P. H. et al. Bone formation in vivo: comparison of osteogenesis by transplanted mouse and human marrow stromal fibroblasts. Transplantation $\mathbf{6 3}$, 1059-1069 (1997).

33. Monfoulet, L., Rabier, B., Chassande, O. \& Fricain, J. C. Drilled hole defects in mouse femur as models of intramembranous cortical and cancellous bone regeneration. Calcif. Tissue Int. 86, 72-81 (2010).

34. He, Y. et al. p53 loss increases the osteogenic differentiation of bone marrow stromal cells. Stem Cells 33, 1304-1319 (2015).

35. Koide, $\mathrm{H}$. et al. Mice deficient in AKAP13 (BRX) are osteoporotic and have impaired osteogenesis. J. Bone Min. Res. 30, 1887-1895 (2015).

36. Agata, $\mathrm{H}$. et al. Characteristic change and loss of in vivo osteogenic abilities of human bone marrow stromal cells during passage. Tissue Eng. Part A 16, 663-673 (2010).

37. Liu, S. et al. Manufacturing differences affect human bone marrow stromal cell characteristics and function: comparison of production methods and products from multiple centers. Sci. Rep. 7, 46731 (2017).

38. Kuznetsov, S. A. et al. Single-colony derived strains of human marrow stromal fibroblasts form bone after transplantation in vivo. J. Bone Miner. Res. 12, 1335-1347 (1997).

39. Satoh, W., Gotoh, T., Tsunematsu, Y., Aizawa, S. \& Shimono, A. Sfrp1 and Sfrp2 regulate anteroposterior axis elongation and somite segmentation during mouse embryogenesis. Development 133, 989-999 (2006).

40. Rattner, A. et al. A family of secreted proteins contains homology to the cysteinerich ligand-binding domain of frizzled receptors. Proc. Natl. Acad. Sci. U.S.A. 94, 2859-2863 (1997).

41. Lopez-Rios, J., Esteve, P., Ruiz, J. M. \& Bovolenta, P. The Netrin-related domain of Sfrp1 interacts with Wnt ligands and antagonizes their activity in the anterior neural plate. Neural Dev. 3, 19 (2008).

42. Bovolenta, P., Esteve, P., Ruiz, J. M., Cisneros, E. \& Lopez-Rios, J. Beyond Wnt inhibition: new functions of secreted Frizzled-related proteins in development and disease. J. Cell Sci. 121, 737-746 (2008).

43. Bafico, A. et al. Interaction of frizzled related protein (FRP) with Wnt ligands and the frizzled receptor suggests alternative mechanisms for FRP inhibition of Wnt signaling. J. Biol. Chem. 274, 16180-16187 (1999).

44. Uren, A. et al. Secreted frizzled-related protein-1 binds directly to Wingless and is a biphasic modulator of Wnt signaling. J. Biol. Chem. 275, 4374-4382 (2000).

45. Rodriguez, J. et al. SFRP1 regulates the growth of retinal ganglion cell axons through the Fz2 receptor. Nat. Neurosci. 8, 1301-1309 (2005).
46. von Marschall, Z. \& Fisher, L. W. Secreted Frizzled-related protein-2 (sFRP2) augments canonical Wnt3a-induced signaling. Biochem. Biophys. Res. Commun. 400, 299-304 (2010).

47. Alfaro, M. P. et al. sFRP2 suppression of bone morphogenic protein (BMP) and Wnt signaling mediates mesenchymal stem cell (MSC) self-renewal promoting engraftment and myocardial repair. J. Biol. Chem. 285, 35645-35653 (2010).

48. Misra, K. \& Matise, M. P. A critical role for sFRP proteins in maintaining caudal neural tube closure in mice via inhibition of BMP signaling. Dev. Biol. 337, 74-83 (2010).

49. Oshima, T. et al. Myeloma cells suppress bone formation by secreting a soluble Wnt inhibitor, sFRP-2. Blood 106, 3160-3165 (2005).

50. Boland, G. M., Perkins, G., Hall, D. J. \& Tuan, R. S. Wnt 3a promotes proliferation and suppresses osteogenic differentiation of adult human mesenchymal stem cells. J. Cell. Biochem. 93, 1210-1230 (2004).

51. Gaur, T. et al. Canonical WNT signaling promotes osteogenesis by directly stimulating Runx2 gene expression. J. Biol. Chem. 280, 33132-33140 (2005).

52. Nakanishi, R. et al. Osteoblast-targeted expression of Sfrp4 in mice results in low bone mass. J. Bone Min. Res. 23, 271-277 (2008).

53. Li, G. et al. SFRP2 promotes stem cells from apical papilla-mediated periodontal tissue regeneration in miniature pig. J. Oral. Rehabil. 47(Suppl 1), 12-18 (2020).

54. $\mathrm{Yu}, \mathrm{G}$. et al. Demethylation of SFRP2 by histone demethylase KDM2A regulated osteo-/dentinogenic differentiation of stem cells of the apical papilla. Cell Prolif. 49, 330-340 (2016).

55. Robey, P. G. Cell sources for bone regeneration: the good, the bad, and the ugly (but promising). Tissue Eng. B Rev. 17, 423-430 (2011).

56. Stuart, T. et al. Comprehensive integration of single-cell data. Cell 177, 1888-1902 e1821 (2019).

57. Bouxsein, M. L. et al. Guidelines for assessment of bone microstructure in rodents using micro-computed tomography. J. Bone Min. Res. 25, 1468-1486 (2010).

58. de Castro, L. F. et al. Role of the N- and C-terminal fragments of parathyroidhormone-related protein as putative therapies to improve bone regeneration under high glucocorticoid treatment. Tissue Eng. Part A 16, 1157-1168 (2010).

59. Gage, G. J., Kipke, D. R. \& Shain, W. Whole animal perfusion fixation for rodents. J. Vis. Exp. 65, 3564 (2012).

60. Kuznetsov, S. A., Friedenstein, A. J. \& Robey, P. G. Factors required for bone marrow stromal fibroblast colony formation in vitro. Br. J. Haematol. 97, 561-570 (1997).

61. Robey, P. G., Kuznetsov, S. A., Riminucci, M. \& Bianco, P. Bone marrow stromal cell assays: in vitro and in vivo. Methods Mol. Biol. 1130, 279-293 (2014).

62. Suresh, S., de Castro, L. F., Dey, S., Robey, P. G. \& Noguchi, C. T. Erythropoietin modulates bone marrow stromal cell differentiation. Bone Res. 7, 21 (2019).

(i) Open Access This article is licensed under a Creative Commons Attribution 4.0 International License, which permits use, sharing, adaptation, distribution and reproduction in any medium or format, as long as you give appropriate credit to the original author(s) and the source, provide a link to the Creative Commons license, and indicate if changes were made. The images or other third party material in this article are included in the article's Creative Commons license, unless indicated otherwise in a credit line to the material. If material is not included in the article's Creative Commons license and your intended use is not permitted by statutory regulation or exceeds the permitted use, you will need to obtain permission directly from the copyright holder. To view a copy of this license, visit http://creativecommons. org/licenses/by/4.0/.

(c) The Author(s) 2021 